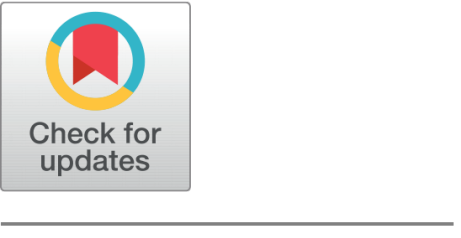

OPEn ACCESS

Received: 29.08 .2020

Accepted: 24.09 .2020

Published: 05.10 .2020

Editor: Dr. Natarajan Gajendran

Citation: Ravikumar K, Ithaya Priya B (2020) Identification of multiple crack locations in micro cantilever beam by wavelet transforms. Indian Journal of Science and Technology 13(35): 3685-3694. https://doi.org/ 10.17485/IJST/v13i35.1472

*Corresponding author. priyatrp25@gmail.com

Funding: None

Competing Interests: None

Copyright: ( $) 2020$ Ravikumar \& Ithaya Priya. This is an open access article distributed under the terms of the Creative Commons Attribution License, which permits unrestricted use, distribution, and reproduction in any medium, provided the original author and source are credited.

Published By Indian Society for Education and Environment (iSee)

ISSN

Print: 0974-6846

Electronic: 0974-5645

\section{Identification of multiple crack locations in micro cantilever beam by wavelet transforms}

\author{
K Ravikumar ${ }^{1}$, B Ithaya Priya ${ }^{2 *}$ \\ 1 Engineering Mathematics, Faculty of Engineering and Technology, Annamalai University, \\ Annamalai Nagar, 608002, Tamil Nadu, India \\ 2 Department of Mathematics, Annamalai University, Annamalai Nagar, 608002, Tamil Nadu, \\ India
}

\section{Abstract}

Background/Objectives: Presence of cracks in micro cantilever beams badly influence the performance of the device. This study is to propose suitable method utilizing wavelet transform to detect the cracks present over the structure of micro cantilever beams. Methods: The proposed device structure was designed and modeled using COMSOL Multi-physics simulation software. The signals obtained are analyzed using wavelet transform. Findings: Micro cantilevers shows difference in its vibrational frequency, stress and deflection over various input conditions. The location of the multiple cracks which appears in micro cantilever structure due to various working conditions were perfectly identified using wavelet transform analysis. Novelty/Applications: We report the accurate locations of the crack present in micro structured devices. This ideal method can be applied on micro cantilever devices which found its applications as Transducers, Sensors, Switches, Actuators and Probes.

Keywords: Wavelet analysis; crack detection; cantilever beam; MEMS; COMSOL

\section{Introduction}

Wavelet transform is one among the effective signal processing techniques based on a windowing approach of dilated scaled and shifted wavelets which is being applied to a broad range of engineering applications ${ }^{(1)}$. An effective signal processing techniques should have potential to analyze continuous and transient signals in both time and frequency domains. But Fourier transform can provide efficient signal analysis in frequency domain, but it is inappropriate for time varying signals due to unavailability of local data. Wavelet transform represents to be an efficient and alternative method to Fourier transform, since it is based on a windowing technique with variable sized region. Wavelet transform has the ability to provide good time and frequency resolutions of a signal $^{(2)}$. This instinctive feature of wavelet transform made it very popular for various engineering applications.

The concept of wavelet was first proposed by Jean Morlet in 1980 by constructing nontrivial wavelets which are continuously differentiable. Later Mayer, Daubachies and Mallet changed this by defining the wavelets for various applications like time frequency 
spectral analysis, digital signal processing, data compression and image processing. According to Mallet, there are two types of wavelet functions in performing wavelet transforms, real wavelets and analytical wavelets ${ }^{(3)}$. The real wavelets are those wavelets used to detect sharp signal transitions and analytic wavelets are used to identify rapid frequency evolution.

In this present work, we proposed a distinctive method by utilizing the wavelet coefficients to analyze the output signal from a micro structured cantilever beam to locate any damages or cracks present ${ }^{(4)}$. The damage detection can be obtained by sharp transitions of signals due to change in structural dynamics ${ }^{(5)}$. The wavelet coefficients will reflect with peaks when any changes or discontinuities occur in data. By the use of such ridges and peaks in wavelet analysis, we could exactly locate the cracks and damages present in the micro cantilever beam ${ }^{(6,7)}$. Dynamic characteristics of the beam will reflect with respect to the damage or cracks over the surface. Here we had compared devices with various dissimilarities in the device surface. The device with structural damages is compared with healthy device and the presence of location is identified by use of wavelet transform analysis.

\section{Wavelet analysis}

Wavelet transform was utilized to investigate the complex signals in both time and frequency domain. A wavelet is a wave like structure with amplitude which begins from zero to maximum and then decreases to zero. When a signal represented by a function $\mathrm{f}(\mathrm{t})$ is studied, the wavelet transform is defined as shown in equation 1 ,

$$
W_{f(a, b)}=\int_{-\infty}^{\infty} f(t) \Psi_{a, b}(t) d t
$$

Where the parameters a, b represents the time and frequency scale respectively. $\Psi(t)$ is called the mother wavelet and $\Psi_{a, b}(t)$ is a scaled and translated wavelet as equation 2 ,

$$
\begin{gathered}
\Psi_{a, b}(t)=\frac{1}{\sqrt{a}} \Psi\left(\frac{t-b}{a}\right) \\
\therefore W_{f(a, b)}=\frac{1}{\sqrt{a}} \int_{-\infty}^{\infty} f(t) \Psi\left(\frac{t-b}{a}\right) d t
\end{gathered}
$$

Wavelet transform is classified into continuous wavelet transform(CWT) and discrete wavelet transform(DWT). CWT is used to provide the complete representation of signal with respect to the mother wavelet which vary continuously, definition of CWT is given in equation 4 ,

$$
C W T_{(a, b, \Psi)}=W \int_{-\infty}^{\infty} x(t) \Psi^{*}\left(\frac{t-b}{a}\right) d t
$$

Where ${ }^{*}$ denotes the complex conjugate, $\Psi$ is wavelet function, $\mathrm{x}(\mathrm{t})$ is the signal in time domain and W is weighting function. Poisson wavelet, Morlet wavelet, Shannon wavelet, Meyer wavelet and Mexican hat wavelet are some of the commonly used mother wavelets in CWT.

DWT are often used where the signals are broken into discrete blocks. In this type of transform the wavelets are discretely sampled. DWT is derived by critical sampling of CWT with greater reduction in the number of dilations and shifts. The wavelet can be constructed from a scaling function and must be orthogonal to its discrete translations. The wavelet is obtained from scaling function shown in equation 5 ,

$$
\psi(x)=\sum_{k=-\infty}^{\infty}-1^{k} a_{n-1-k} \psi(2 x-k)
$$

Haar wavelet was the first DWT invented which is a sequence of rescaled square shaped functions which together form a wavelet $^{(8,9)}$. Haar wavelet is the only symmetric compactly supported orthogonal wavelet. Haar mother wavelet function is defined as,

$$
\psi(t)=\left\{\begin{array}{cc}
1 & 0 \leq t \leq \frac{1}{2} \\
-1 & \frac{1}{2} \leq t<1
\end{array}\right.
$$


Its scaling function $\varphi(t)$ can be described as

$$
\varphi(t)= \begin{cases}1 & 0 \leq t<1 \\ 0 & \end{cases}
$$

Daubachies wavelet represents the collection of wavelets which are localized in both frequency domain and spatial domain. Here all basic functions are mutually orthogonal and normalized. The scaling functions and wavelets are defined recursively through the use of dilation equation. Daubachies wavelets have different orders which are represented by $\mathrm{N}$, which ranges from $\mathrm{db} 1$ to $\mathrm{dbN}$. Where, db1 wavelet coefficient is almost same and equal to Haar wavelet. Daubechies wavelets found to perform well in such applications due to its proven effectiveness in this field ${ }^{(10)}$. Here both orthogonal and bi-orthogonal wavelet functions were used to detect the location and characteristics of the cantilever beam ${ }^{(11)}$.

Here for our study we had analyzed the signal along the axis of the beam using DWT. We use the mother wavelets like Bior, Haar and Daubachies wavelets for locating the cracks present in the micro cantilever beam. Information about the cracks was obtained by the discreet wavelet transform (DWT) coefficients.

\section{Micro Cantilever Beam}

Cantilever beam is a mechanical structure fixed at one end and free at the other end. When a cantilever is subjected to a load, it will bend to extend due to the load and stress will be generated over the structure due to its deflection. Micro cantilever beam whose dimension will be in micrometer scale will result with large displacement for small change in mass, force or stress. Micro cantilevers are primarily used in sensors, switches, actuators and transducers ${ }^{(12)}$. Micro cantilever based sensor is one of the most commonly used mechanical sensor. Cantilever devices are used as transducer which converts mechanical behavior of cantilever into measurable signals. Physical changes occurred in the devices due to external disturbances or environmental changes effects the mechanical behavior. Micro cantilever based devices found its application in gas, temperature, pressure and biological sensors ${ }^{(13)}$. Its reduced micro scale dimension will make them more sensitive, cost effective and faster. It can be implanted over any surface due to its reduced dimension and compatibility. Bulk micromachining and surface micromachining are the most efficient fabrication techniques in fabricating such micro devices. The materials such as silicon, gold and platinum are widely used for fabrication due to its selectivity, sensitivity and ease of processing technology. The basic structure of the micro cantilever beam is shown in the Figure 1, where $\mathrm{L}$ represents the length of the beam, $\mathrm{W}$ is the beam width and $\mathrm{T}$ is the thickness of the beam. Bending or deflection caused in the beam can produce differential stress over the surface. These devices are one among the efficient tool for sensing application due to its intrinsic flexibility. The sensing action is based on deflection of the beam and the stress developed due to external force ${ }^{(14)}$.

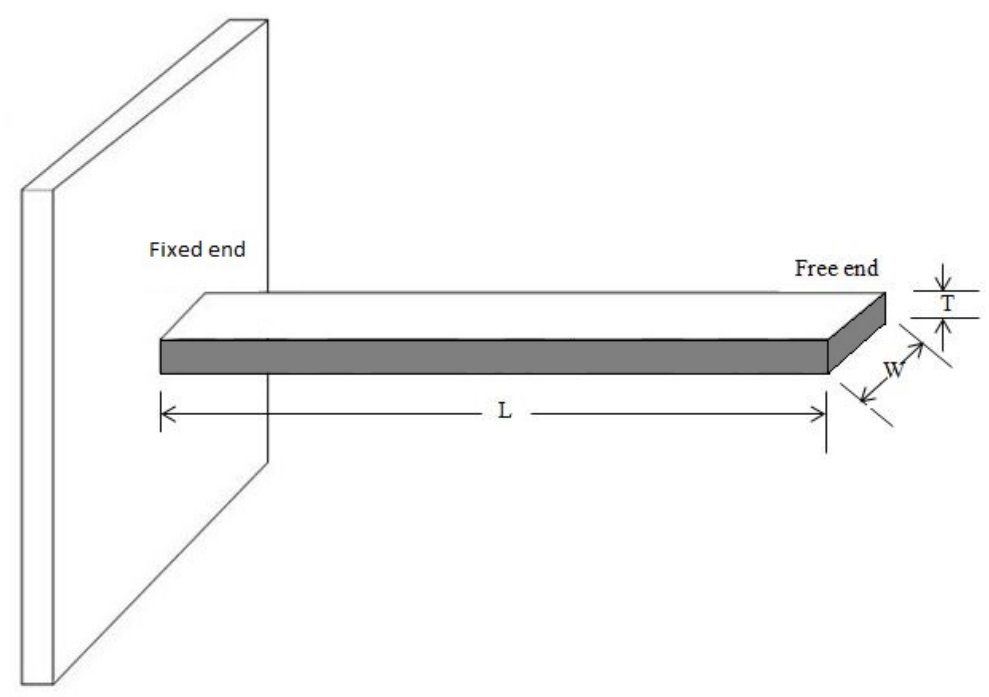

Fig 1. Basic cantilever beam structure

Since the device is sensitive for very small input changes it is necessary to monitor the device health to get efficient output. Even a small damage or crack present in the device structure due to fabrication process or environmental conditions will reflect 
in the output to a greater extent ${ }^{(15)}$. In this work we aim to identify the presence of cracks present in the device structure by analyzing the output signal of the measured device compared to the healthy cantilever beam with respect to the stress and total displacement. Figure 2 shows the SEM images of cracks possibilities in the micro cantilever structure which was reported earlier.
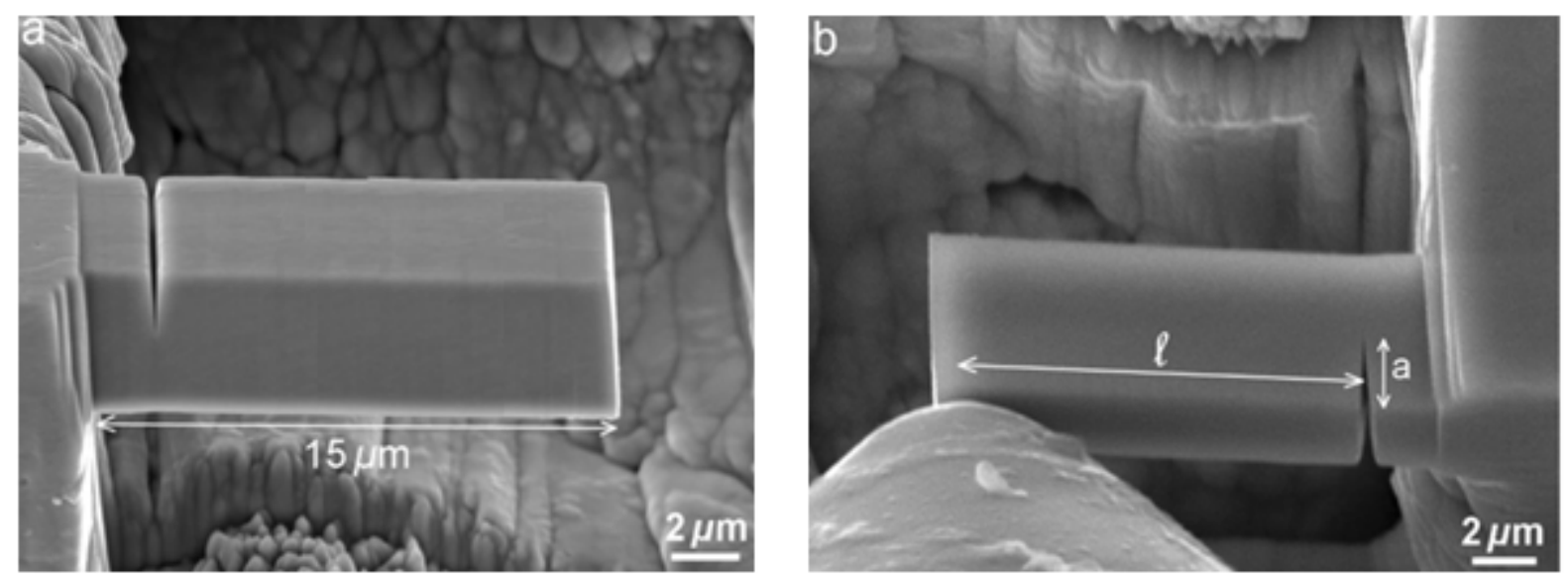

Fig 2. SEM image of micro cantilever beam with cracks

\section{Modeling of Micro Cantilever Beam structure}

In our work, the structure of proposed micro cantilever is designed using COMSOL multiphysics5.4 simulation software. Computer simulation is very essential in developing new products and for optimization of particular design. COMSOL is one which helps to develop and determine the accuracy of the model with real world precision. The model can be tested over various geometrical characteristic and physical parameters. Defining the geometries, material property and physics of the model can be encompassed with step by step modeling workflow. COMSOL multiphysics5.4 brings enhanced modeling features for modeling thin layered structure. Any combination of add on modules for various engineering applications can be simulated using this tool.

In this proposed study, we had chosen poly crystalline silicon as device material due to its unique properties in fabricating micro structured devices. Material properties of Poisson ratio -0.22 , Density $-2320 \mathrm{~kg} / \mathrm{m}^{3}$ and youngs modulus-160Gpa is chosen. Next the device parameters of length (L) $150 \mu \mathrm{m}$, width (W) $20 \mu \mathrm{m}$ and thickness (T) $2 \mu \mathrm{m}$ were fixed. The force (P) applied over the entire beam is $3.5 \times 10^{-6} \mathrm{~N}$. For the above mentioned, material property and device dimensions, we calculate the deflection of the device theoretically using the equation 6 ,

$$
\delta=\frac{P L^{3}}{24 E I} \frac{x^{2}}{L^{2}}\left(6-4 \frac{x}{L}+\frac{x^{2}}{L^{2}}\right)
$$

Where, $\mathrm{x}$ is the specific point the deflection to be calculated. We consider $\mathrm{x}=\mathrm{L}$ for calculating the maximum deflection of the cantilever beam.

$$
\delta_{\text {max }}=\frac{P L^{3}}{8 E I}
$$

Where, $\mathrm{E}$ is the young modulus of the material, I is the moment of inertia, $\mathrm{P}$ the force applied and $\mathrm{L}$ is the beam length, $\mathrm{W}$-width, T-thickness. Moment of inertia is given as equation 8 ,

$$
I=\frac{1}{12} W \cdot T^{3}
$$

From the above equations, we calculated the theoretical value of maximum deflection to be $\delta_{\max }=0.687 \mu \mathrm{m}$. For the purpose of locating cracks present in the device surface, we compared three devices with different cracks which were intentionally made over the device structure. The constant crack dimensions of length $\mathrm{L}=0.5 \mu \mathrm{m}$, width $\mathrm{W}=10 \mu \mathrm{m}$ were made over all the three devices. Figure 3 shows the schematic diagram of cantilever beam with crack in its surface. Table 1 gives the details of cracks present in the respective devices. 


\section{Force}

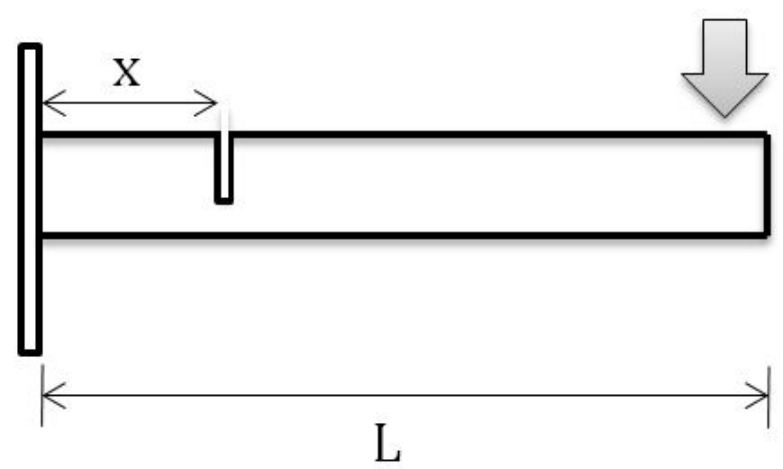

Fig 3. Schematic Cantilever Beam with single crack

Table 1. Device details with crack locations

\begin{tabular}{llll}
\hline Sl.no & Device & No. of Cracks Present & Location of the cracks in $\mathrm{x}$ axis \\
\hline 1 & D & Nil & Reference Healthy Beam \\
2 & D1 & 1 & $30 \mu \mathrm{m}$ \\
3 & D2 & 2 & $30 \mu \mathrm{m} \& 60 \mu \mathrm{m}$ \\
\hline
\end{tabular}

\section{Simulation Result and Discussions}

\subsection{Deflection Analysis}

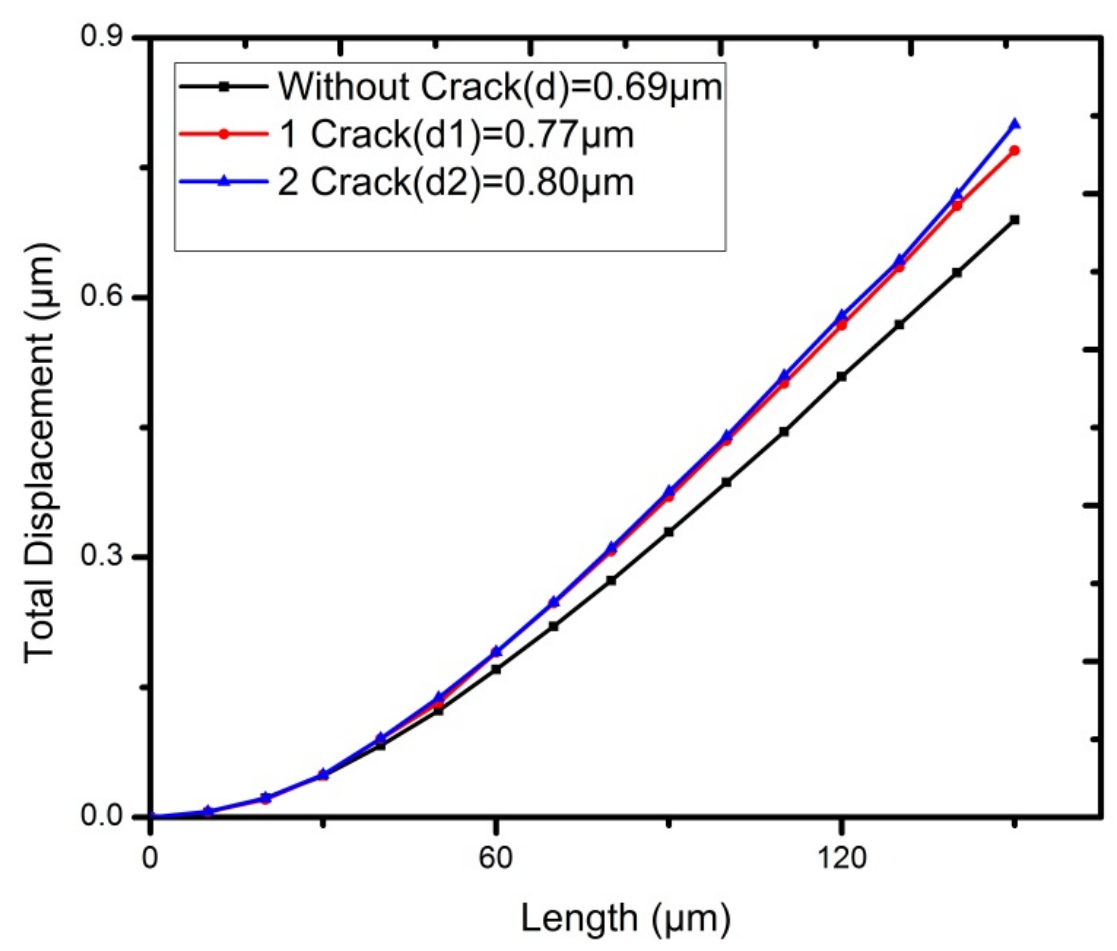

Fig 4. Comparison of Total Displacement for the devices 
When a force is applied over the beam surface it will deflect to all extend with respect to the force applied. Here in our study we applied a constant force of $3.5 \times 10^{-6} \mathrm{~N}$. The force is kept constant for all our structures in order to compare the device characteristics. The micro cantilever beam $\mathrm{D}$ without any crack is assumed to be the healthy device, shows maximum deflection of $0.69 \mu \mathrm{m}$ for above mentioned force. This maximum deflection of this device is almost equal to our theoretical value calculated which justifies our device modelling. The device $\mathrm{D}_{1}$ with single crack show deflection of $0.77 \mu \mathrm{m}$ total displacement. The occurrence of crack makes the beam deflect more than the healthy structure due to the damage present in the device structure. Device $\mathrm{D}_{2}$ with two cracks deflects more than the above two structures with total displacement $0.80 \mu \mathrm{m}$. The number of cracks in the device structure makes their impact over the total displacement of the device. The deflection of the beam increases with respect to the cracks over its surface. From the above analysis, we conclude the device with maximum cracks will deflect more than other device structures.

Figure 4 shows the deformation of beam structure for three types in terms of total displacement. The difference in deflection between the device $\mathrm{D}_{2}$ and $\mathrm{D}_{3}$ is very closer, since it depends upon the crack dimension and its location. But healthy structure and cracked beams shows conspicuous displacement.

\subsection{Stress Analysis}

When a cantilever beam is subjected to load it will deflect as the result the stress will be developed as force gets distributed over the entire structure. Usually the stress will be concentrated on the fixed end as the entire load will be accumulated at the fixed end and gradually decreases towards the free end. But in case of cracked structure, the stress will be maximum at the cracked point and then distributed over the surface. Figure 5 shows the pictorial representation and one dimensional view of stress over healthy micro cantilever structure obtained through COMSOL simulation software. The 1D plot describes the stress over the surface. The stress is maximum at the fixed end of the beam then decreases gradually towards free end. Figure 6 shows the pictorial representation and one dimensional view of stress over the micro cantilever beam with one crack at $30 \mu \mathrm{m}$ from fixed end. It clearly shows the peak values of stress across the cracked region of the device. The pictorial representation of stress and one dimensional plot for device with two cracks where given in the Figure 7 where the cracked regions of device structure deflects with peak. The stress is found to be maximum in the crack present at $30 \mu \mathrm{m}$ which is near the fixed end when compare to the other crack at $60 \mu \mathrm{m}$.
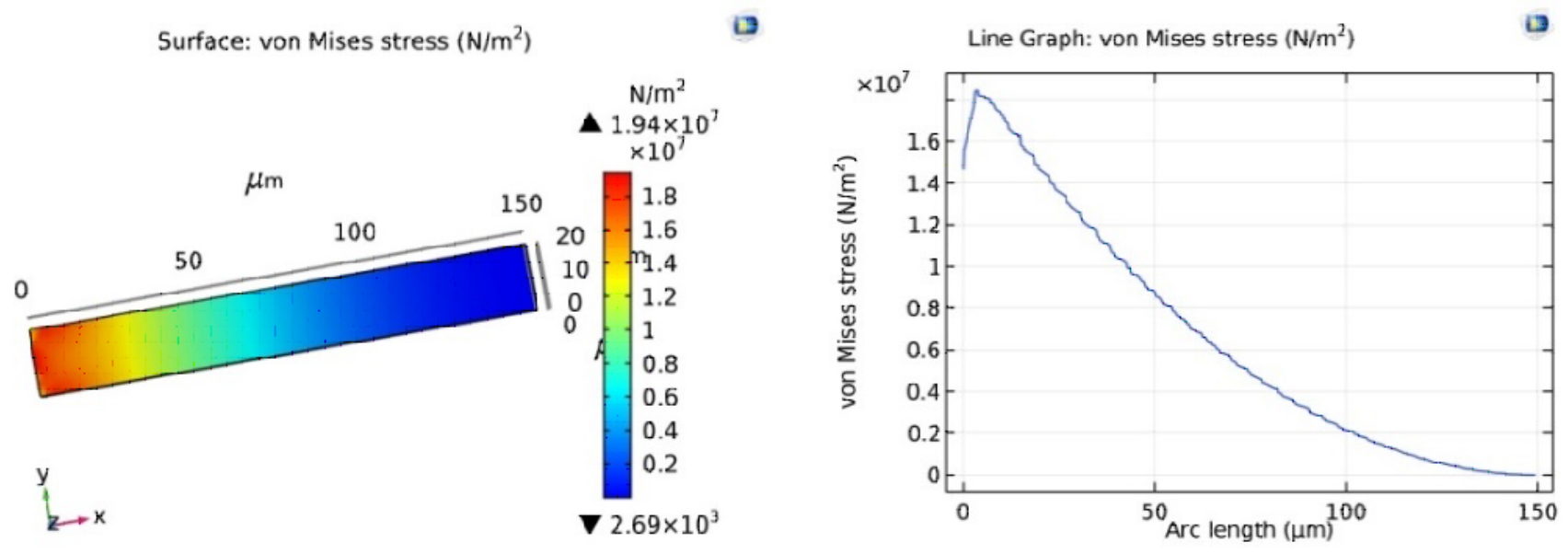

Fig 5. Pictorial and 1D representation of Healthy beam 

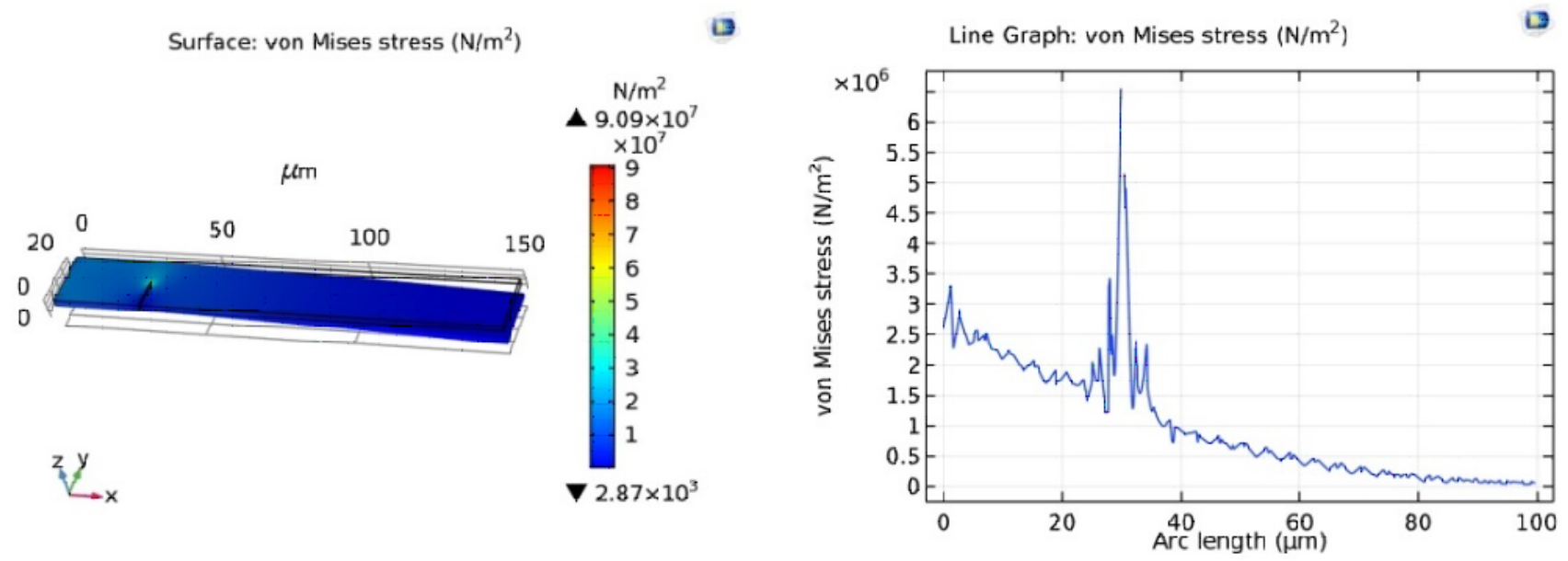

Fig 6. Pictorial and $1 \mathrm{D}$ representation of beam with $1 \mathrm{crack}$

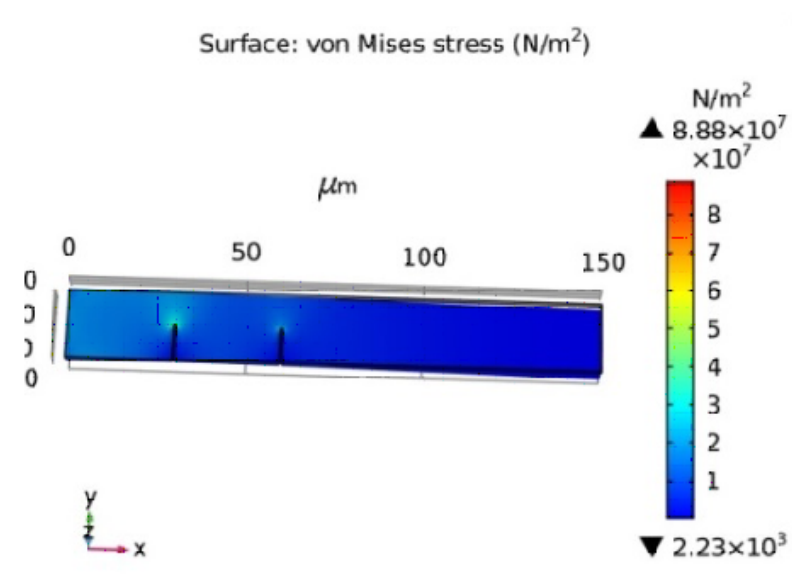

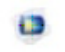

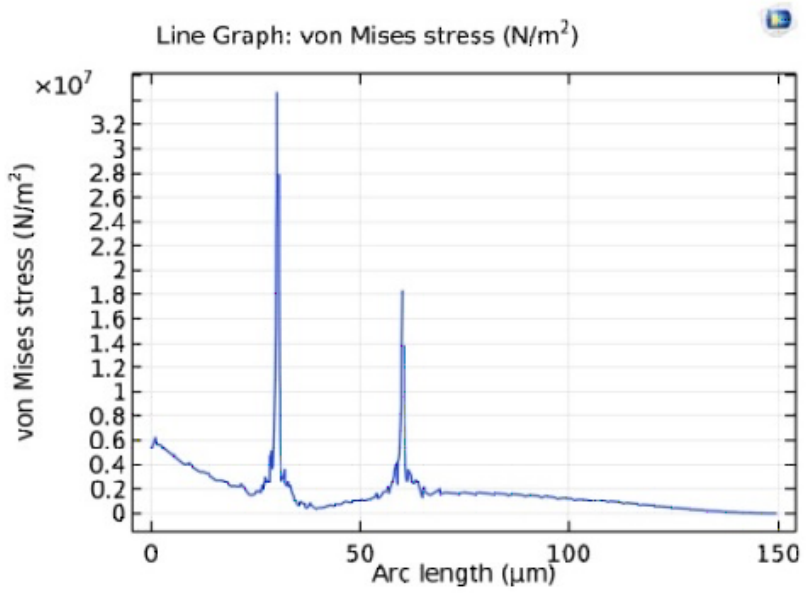

Fig 7. Pictorial and $1 \mathrm{D}$ representation of beam two cracks

\subsection{Analysis using Wavelet transforms}

The data obtained using COMSOL simulation is analyzed using orthogonal and bi-orthogonal wavelet coefficient with various mother wavelets like Bior3.5, Haar and Daubechies. Selection of efficient wavelet functions is usually done by trial and error method. Symmetric wavelets were selected for application like crack detection. The change in wavelet coefficients with peaks and ridges due to discontinuity of signals were obtained. The Figure 8 represents the wavelet coefficients towards the stress obtained for healthy micro cantilever beam using different mother wavelets. The wavelet coefficient shows peaks near the fixed end where the stress is to be high and reduces gradually along the axis of the beam. Here the beam length of $150 \mu \mathrm{m}$ is differentiated to 250 nodes and the analysis was carried out. The Figure 9 shows the wavelet response for the stress over the surface of the beam with crack at $30 \mu \mathrm{m}$. Here the wavelet coefficients shows peak exactly only at the crack position and retained to be normal where the stress will be negligible. Here the beam length is differentiated to 250 nodes for wavelet analysis.

Figure 10 shows the wavelet transform of cantilever beam with two cracks for different mother wavelet coefficients. The length of the beam is differentiated to 600 nodes and the wavelet coefficients respond perfectly where the cracks present. The crack present at $30 \mu \mathrm{m}$ experiences higher stress then the crack present at $60 \mu \mathrm{m}$ which is very clearly revealed by the wavelet response. Among these different mother wavelets, Daubechies wavelets found to perform well in such applications due to its 
proven effectiveness in this field. Daubechies $\mathrm{db} 4$ and $\mathrm{db} 8$ encodes polynomial with two and four coefficients respectively. This ability to encode the signals is subject to the phenomenon of scale leakage and the lack of shift invariance, which rise from discrete shifting operation during application of the transform. A Daubechies wavelet coefficient shows more sensitivity to the cracks present in the device structure.
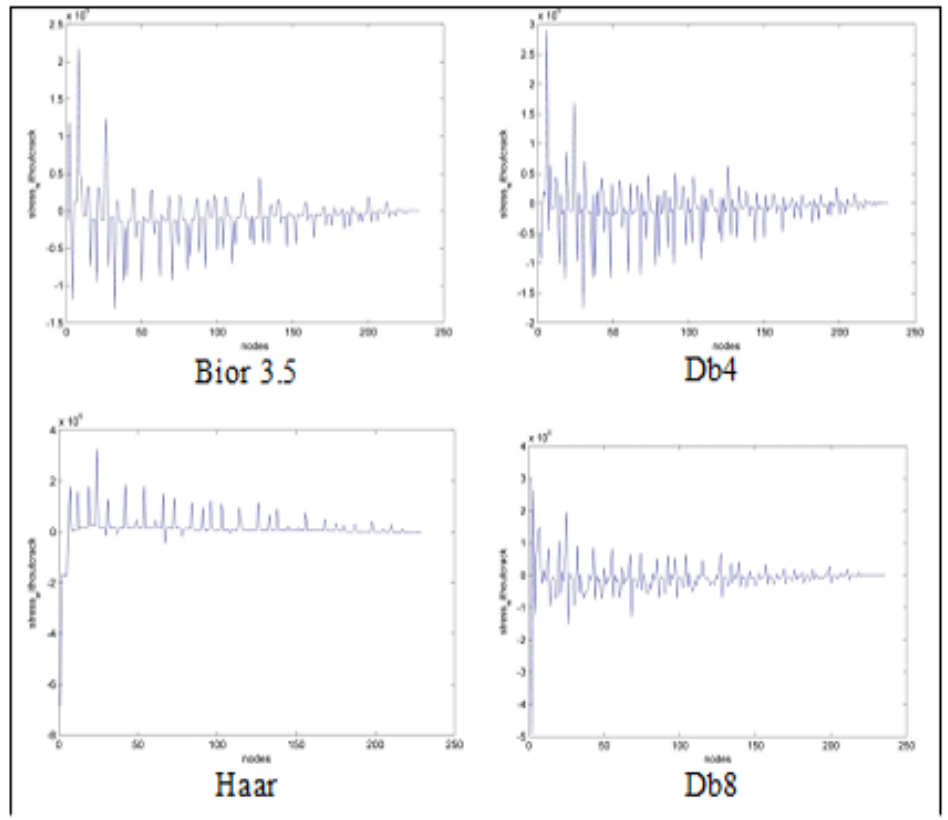

Fig 8. Different wavelet coefficient responses for cantilever beam without crack

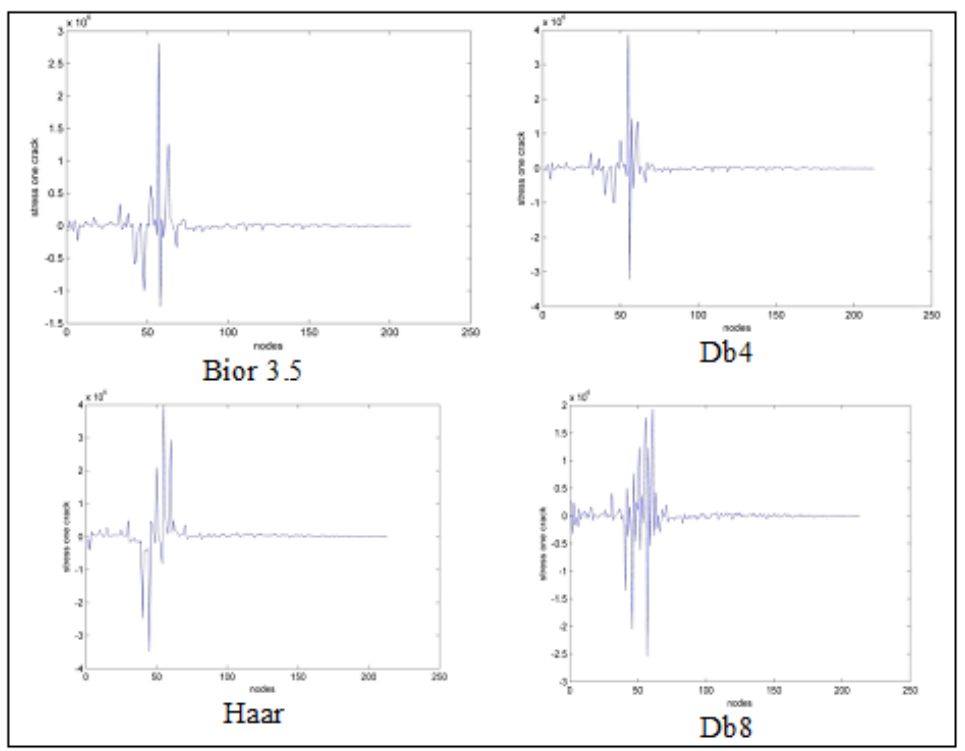

Fig 9. Different wavelet coefficient responses for single crack in cantilever beam 


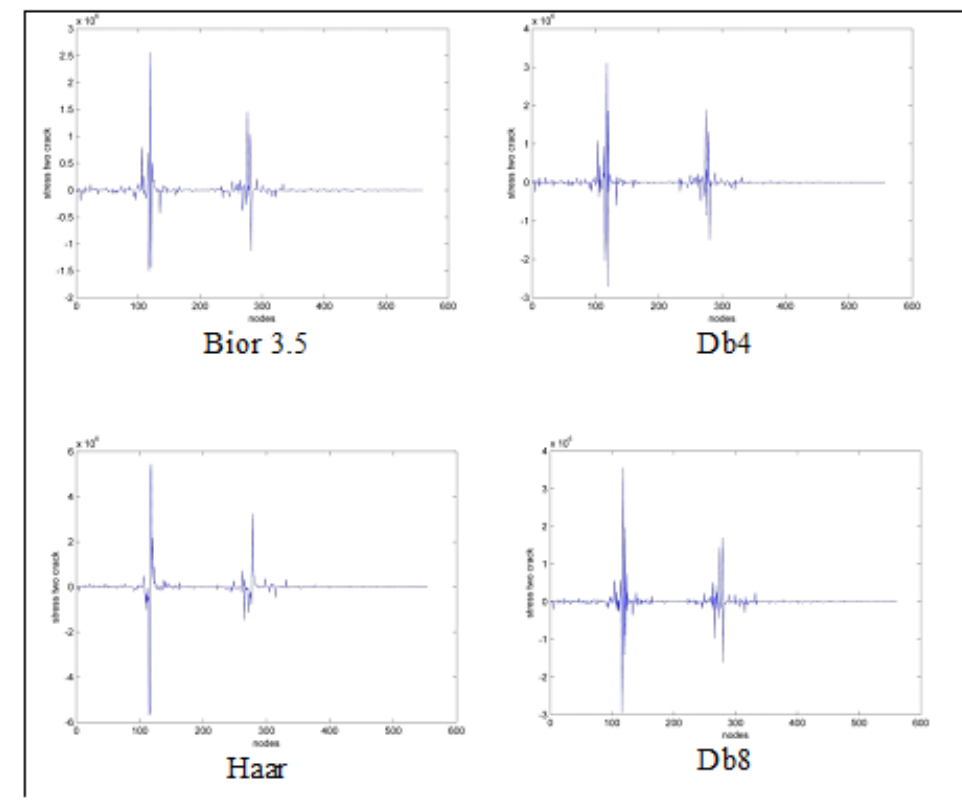

Fig 10. Different wavelet coefficient responses for cracks at various locations of cantilever beam

\section{Conclusion}

We proposed a unique method utilizing wavelets in locating the cracks present in micro cantilever beam. The device was modeled using COMSOL simulation software and wavelet transform analysis were carried for the obtained data by MATLAB. The device characteristics like total displacement and stress were examined and compared for different devices. The proposed method describes the effective and efficient methodology to identify the crack location by analyzing the output signals from the device using coefficients of different mother wavelets. Here, Daubechies wavelets respond well with the changes occurred in the device structure and found to be most effective method in crack detection. This novel type approach can be applied for any micro devices for analyzing the damages or any other structural changes occur in the device assembly since it is very sensitive to minute changes.

\section{References}

1) Chui C. An introduction to wavelets, wavelet Analysis and its applications;vol. 1. San Diego. Academic Press. 1992.

2) Sifuzzaman M, Islam M, Ali M. Application of Wavelet Transform and its Advantages Compared to Fourier Transform. J Phys Sci. $2009 ; 13$.

3) Graps A. An introduction to wavelets. IEEE Computational Science and Engineering. 1995;2(2):50-61. Available from: https://dx.doi.org/10.1109/99. 388960.

4) Ravikumar K, Priya BI. Detection of cracks in Micro structured Cantilever Beam using Wavelet Transforms. Ravikumar.K, Ithaya Priya.B. International Journal of Recent Technology and Engineering (IJRTE). 2020;8(6):2277-3878. Available from: https://www.ijrte.org/wp-content/uploads/papers/v8i6/ F7364038620.pdf.

5) Mehta P, Kureshi A, Lad S, Patel N, Sharma D. Detection of Cracks in a Cantilever Beam Using Signal Processing and Strain Energy Based Model. IOP Conference Series: Materials Science and Engineering. 2017;234:012008-012008. Available from: https://dx.doi.org/10.1088/1757-899x/234/1/012008.

6) Ramon SYC, Silva LM, Bezerra. Determination of Damages in Beams using wavelet Transforms. In: and others, editor. Proceedings of the World Congress on Engineering. 2012.

7) \&amp; Jiang YY, \&amp; Li B, Zhang, \&amp; Chen XFZS. Identification of Crack Location in Beam Structures Using Wavelet Transform and Fractal Dimension. Shock and Vibration. 2015. Available from: https://doi.org/10.1155/2015/832763.

8) Akbari J, Ahmadifarid M, Amiri AK. Multiple crack detection using wavelet transforms and energy signal techniques. Frattura ed Integrità Strutturale. 2020;52:269-280. Available from: https://doi.org/10.3221/IGF-ESIS.52.21.

9) Sharif I, Khare S. Comparative Analysis of Haar and Daubechies Wavelet for Hyper Spectral Image Classification. ISPRS - International Archives of the Photogrammetry, Remote Sensing and Spatial Information Sciences. 2014;p. 937-941. Available from: https://doi.org/10.5194/isprsarchives-XL-8-9372014.

10) Cao L, Huiqi L, Yanjun Z, Liang X, Zhang L. Hierarchical method for cataract grading based on retinal images using improved Haar wavelet. Information Fusion. 2019. Available from: https://doi.org/10.1016/j.inffus.2019.06.022.

11) Rousta AM, Beiranvand P, Akbarinia F, Hooshmand M. The Case Examination of Detection of Structural Damages on a Plate using Wavelet Transform". Int J of Advanced Design and Manufacturing Technology. 2020;13(2):91-97. 
12) Rucka M. Damage detection in beams using wavelet transform on higher vibration modes. Journal of Theoretical and Applied Mechanics. $2011 ; 49$.

13) Lucas S, Kis-Sion K, Pinel J, Bonnaud O. Polysilicon cantilever beam using surface micromachining technology for application in microswitches. Journal of Micromechanics and Microengineering. 1999. Available from: https://doi.org/10.1088/0960-1317/7/3/021.

14) Jiang Y, Wang N, Zhong Y. A two-step damage quantitative identification method for beam structures. Measurement. 2021. Available from: https: //dx.doi.org/10.1016/j.measurement.2020.108434.

15) Lee Y, Liew Y, K. Detection of damage location in a beam using the wavelet analysis. International Journal of Structural Stability and Dynamics. 2012. Available from: https://doi.org/10.1142/S0219455401000238. 